value of $\geq 200 \mu \mathrm{g} / \mathrm{g}$ was used to indicate active disease. The cohort was subdivided into 4 groups: active and inactive Crohn's disease (CD), active and inactive Ulcerative colitis (UC).

Results 1226 patients (771 CD, F=65\%, 455 UC, F = 35\%) with matched FC and FBC data were analysed. The median age was $44 \mathrm{y}$ (IOR 31-57), median disease duration 102 months (IOR 31-207). Overall, $314 / 1226$ patients (25.6\%) were anaemic, 185/314 (58.9\%) of which were female. Anaemia was observed more frequently in patients with active as opposed to inactive CD $(110 / 328$ [33.5\%] vs $65 / 443[14.7 \%], \mathrm{p}<0.0001)$. This pattern was also seen in patients with UC $(129 / 293$ [44\%] vs 23/162 [14.2\%], p < 0.0001). The prevalence of anaemia in active UC was greater than in active CD $(p=0.014)$; however, this could be explained by the higher median FC in the UC cohort (900 vs. 618, $p<0.0001)$. There was no statistically significant difference in age or Montreal location (L1 + L3 vs $\mathrm{L} 2, \mathrm{p}=0.16$ ) between the groups. ROC analysis of FC as a predictor of anaemia showed an AUC of 0.69 with a sensitivity and specificity of 0.73 and 0.57 respectively at a cut off of $200 \mu \mathrm{g} / \mathrm{g}$.

Conclusion In this cohort over $25 \%$ of patients with IBD were anaemic. There is a clear correlation between disease activity and anaemia in both CD and UC, but this is unrelated to disease distribution in CD. Anaemia in asymptomatic patients should alert clinicians to the possibility of subclinical active mucosal inflammation. These data and the ROC analysis provide further support for optimising disease treatment in IBD, targeting a FC level of $<200 \mu \mathrm{g} / \mathrm{g}$. Disclosure of Interest None Declared.

\section{PWE-095 A 10 YEAR REVIEW OF THE DEATH RATE AND CAUSE OF DEATH WITHIN A DISTRICT GENERAL COHORT OF INFLAMMATORY BOWEL DISEASE PATIENTS}

doi:10.1136/gutjnl-2013-304907.383

1."M W Johnson, ' $\mathrm{K}$ Lithgo, 'T Prouse, 'T Price. ' $G$ astroenterology, Luton \& Dunstable University Hospital, Luton, UK

Introduction Whilst there is a growing body of data supporting the increased risk of colo-rectal cancer in patients with inflammatory bowel disease, little has been written about the overall mortality and cause of death seen in patients with this condition.

Objective To assess the death rate and cause of death in our cohort of IBD patients.

Methods A database of all known local IBD patients was established after retrospectively reviewing 37,000 colonoscopy and flexible sigmoidoscopy reports performed at the Luton \& Dunstable University Hospital between 2001 and 2011. Histological assessment was then used to cross correlate all patients diagnosed with colitis. The hospital coding officer analysed the database and provided details on all deaths within that cohort over the time period and the cause of death as listed by the hospital record system.

Results In total 2640 patients with IBD were identified and 186 (7\%) of these died between 2001 and 2011. The average age of death was 80 years. The cause of death ranged considerably (see chart).

\section{Abstract PWE-095 Table}

\begin{tabular}{lll}
\hline Cause of Death & Number & Percentage (\%) \\
\hline Pneumonia & 32 & 17.2 \\
Sepsis & 28 & 15.1 \\
Cardiac & 22 & 11.8 \\
Non-GI Cancer & 11 & 5.9 \\
Crohn's disease & 11 & 5.9 \\
Ulcerative colitis & 9 & 4.8 \\
GI Cancer & 9 & 4.8 \\
Stroke & 8 & 4.3 \\
GI Haemorrhage & 6 & 3.2 \\
\hline
\end{tabular}

Conclusion The Office for National Statistics stated that in 2010 the average lifespan in England and Wales, for men and women, was 85 and 89 years, respectively. The life expectancy in Luton is slightly lower than the national average by approximately 2 years (Annual Public Health Report 2012-2013). The average age of death in our IBD cohort appears substantially lower than expected, with just 41 of the 186 $(22 \%)$ being related to gastrointestinal causes. Infection (sepsis and pneumonia) appeared to be the single most common cause of mortality $60 / 186(37.5 \%)$, raising questions about an iatrogenic contribution.

Disclosure of Interest None Declared.

\section{PWE-096 IS THERE AN ASSOCIATION BETWEEN PARKINSON'S DISEASE AND INFLAMMATORY BOWEL DISEASE ?}

doi:10.1136/gutjin-2013-304907.384

1," $\mathrm{M}$ W Johnson, 'K Lithgo, 'T Price. 'Gastroenterology, Luton \& Dunstable University Hospital, Luton, UK

Introduction The BRAAK theory of Parkinson's disease believes that the aetiology may all start in the bowel with a "slow virus" entering the central nervous system after passing through the intestinal mucosa $\{$ Hawkes C.H., 2007\}. There is already work confirming an increased frequency of $\mathrm{H}$. pylori infections (requiring treatment) in the 5 years prior to Parkinson's disease being diagnosed \{Nielsen H.H. 2012\}. Recently a gene associated with the inherited form of Parkinson's disease (leucine-rich repeat kinase 2 LRRK2), has been shown to regulate the transcription factor NFAT1 (nuclear factor of activated T cells 1), which in turn appears to regulate cells in the immune system, including macrophages, dendritic cells and T cells. Higher quantities of NFAT1 activity are seen in the colonic mucosa of Crohn's patients, where the total quantity directly correlates to the severity of the disease \{Liu Z., 2011\}. Objective: With these theories in mind, we aimed to assess whether a higher than expected association between inflammatory bowel disease (IBD) and Parkinson's disease (PD) could be found.

Methods A cross correlation analysis was performed using the IBD and PD databases at the Luton \& Dunstable University Hospital. A retrospective analysis was also performed using medical notes and the internal electronic results system to assess the disease severity of these two conditions.

Results The prevalence of IBD and PD within the UK population is said to be $225 / 100,000$ (UC 150/100,000 + CrD 75/100,000) and $140 / 100,000$, respectively. The L\&D catchment area covers 330,000 and so one would have expected approximately 742 IBD and 462 PD patients, respectively. The databases had 2783 IBD patients (median age $=51$ ) and $350 \mathrm{PD}$ patients (median age $=79$ ) listed. Probability analysis predicted that we would find just 1 patient with concomitant PK and IBD, however, we found 6 subjects with these conditions concomitantly. This translates into $0.2 \%$ of IBD patients having $\mathrm{PD}$ and $1.72 \%$ of $\mathrm{PD}$ patients having IBD. Mild-to-moderate $\mathrm{PD}$ was noted in 3 patients, and all 3 had mild-to-moderate IBD. Three of the $\mathrm{PD}$ patients were scored as having moderate-to-severe disease, and 2 of these also had moderate-to-severe IBD.

Conclusion The proportion of PD patients having concomitant IBD is considerably higher than one would have expected by chance. This raises possible issues around genetic association, but also lends some credence to theories that PD may owe its origins to the bowel and infective translocation across bowel mucosa. Those patients with more significant IBD also appeared to have more severe PD.

Disclosure of Interest None Declared.

\section{PWE-097 THE EFFECT OF ETHNICITY ON THE PREVALENCE OF INFLAMMATORY BOWEL DISEASE WITH THE LUTON AND DUNSTABLE CATCHMENT REGION, IN UK}

doi:10.1136/gutjnl-2013-304907.385 